\title{
CARTESIAN PRODUCTS OF CONTRACTIBLE OPEN MANIFOLDS
}

BY D. R. McMILLAN, JR.

\author{
Communicated by Edwin Moise, June 27, 1961
}

1. Introduction. In [6], J. H. C. Whitehead gave the first example of a contractible open 3-manifold $W$ topologically different from $E^{3}$. Shapiro and Glimm [2] have shown that $W \times E^{1}$ is topologically $E^{4}$, and Glimm has also noted that $W \times W$ is topologially $E^{6}$. It is the purpose of this note to show that these results hold in general. The unsettled nature of the Poincaré Conjecture for dimension 3 dictates the assumption that each compact subset of the contractible open 3-manifolds considered can be embedded in the 3-sphere $S^{3}$. Such a 3-manifold will be called a $W$-space. The author does not know if each $W$-space can be embedded in $S^{3}$ (see $\$ 4$ ).

The terminology is standard. An $n$-manifold is a countable, connected locally-finite simplicial complex such that the link of each vertex is piecewise-linearly homeomorphic to the usual $(n-1)$-sphere. "Open" is interpreted to mean "without boundary and noncompact," and "closed" to mean "without boundary and compact." All spaces and mappings are taken in the polyhedral or piecewise-linear sense unless stated otherwise. Finally, all the manifolds considered here will be orientable. The author wishes to thank R. H. Bing and David Gillman for their constructive suggestions concerning this paper.

2. Constructing $W$-spaces. All known examples of $W$-spaces can be expressed as the sum of a properly ascending sequence of cubes with handles (i.e., multiple solid tori). Theorem 1 shows this to be a general phenomenon. The author has investigated some examples of $W$ spaces topologically different from Whitehead's first example. In particular, the cubes with handles used in constructing a $W$-space may need to be a genus greater than 1 (as in [3]).

A 3-manifold $M$ with nonempty boundary is irreducible if each loop in Bd $M$ that can be shrunk to a point in $M$ can also be shrunk to a point in $\mathrm{Bd} M$ (e.g., a cube with a knotted tubular hole is irreducible). Otherwise, $M$ is reducible. The following is a consequence of the loop theorem and Dehn's lemma of Papakyriakopoulos or, diectly, of [5].

Lemma 1. $M$ is reducible if and only if there is a disk $D$ in $M$ such that $D \cdot \mathrm{Bd} M=\mathrm{Bd} D$ and $\mathrm{Bd} D$ does not bound a disk in $\mathrm{Bd} M$. 
Let $M$ be a compact 3-manifold with nonempty boundary, and with the property that each closed surface in Int $M$ separates $M$. Then, Lemma 1 and an induction give:

LEMma 2. $M$ contains a finite collection of mutually exclusive disks $\left\{D_{i}\right\}$, such that $D_{i} \cdot \mathrm{Bd} M=\mathrm{Bd} D_{i}$, the closure of each component of $M-\sum D_{i}$ is irreducible, and the closures of any 2 such components are mutually exclusive or their intersection is one of the $D_{i}$ 's. If $M$ has connected boundary then the closure of each component of $M-\sum D_{i}$ has connected boundary.

Let $S$ be a closed surface in the contractible open 3-manifold $U$. Then $U-S$ has precisely 2 components, and the closure of exactly one of these components, called the interior of $S$, is compact. The closure of the interior of $S$ is called the closed interior of $S$. The meaning of the term "interior" should be clear from the context in each case.

THEOREM 1. Let $U$ be a $W$-space. Then, $U=\sum_{i=1}^{\infty} H_{i}$, where each $H_{i}$ is a cube with handles, $H_{i} \subseteq$ Int $H_{i+1}$, and each loop in $H_{i}$ can be shrunk to a point in $H_{i+1}$.

Proof. It clearly suffices to show that each finite polyhedron $P$ in $U$ is contained in the interior of a cube with handles. Since a regular neighborhood of $P$ in $U$ (see [7]) is a compact 3-manifold, and since this 3-manifold is contained in a 3-manifold with connected boundary, the proof of the following will complete the theorem:

If $S$ is a closed surface in $U$, then there is a cube with handles in $U$ whose interior contains the closed interior of $S$.

Let $g$ be the genus of $S$. If $g=0$, the closed interior of $S$ is a 3-cell which can be expanded to give a 3 -cell containing the closed interior of $S$.

Assume the result for surfaces of genus less than $N$, where $N \geqq 1$, and let the genus of the given surface be $N$. Let $C$ be the closed interior of $S$ and let $\left\{T_{j}\right\}$ be 3-cells in $C$ obtained from the disks promised by Lemma 2 for $C$ by a slight thickening. The $T_{j}$ 's are mutually exclusive and each meets $\mathrm{Bd} C$ in an annulus. The closure of each component of $C-\sum T_{j}$ is an irreducible 3 -manifold with connected boundary. Let $B$ denote the set whose components are all of these irreducible 3-manifolds, and let $B^{\prime}$ be the closure of $U-B$. Note that $\mathrm{Bd} B=\mathrm{Bd} B^{\prime}$ and that the collection of surfaces consisting of the components of $\mathrm{Bd} B$ has total genus less than or equal to $N$. If each component of $B$ is a 3-cell then $C$ is a cube with handles and the conclusion follows. 
If not, then there is a simple closed curve $J$ in the interior of a component $B_{0}$ of $B$ such that $J$ cannot be shrunk to a point in $B_{0}$ (one uses here the hypothesis that compact subsets of $U$ can be embedded in $S^{3}$ ). It will now be shown that $B^{\prime}$ is reducible. Let $f$ be a map of a disk $D$ into $U$ such that $f \mid \operatorname{Bd} D$ is a homeomorphism of $\operatorname{Bd} D$ on to $J$ and such that $f$ is in general position with respect to $\mathrm{Bd} B^{\prime}$ in the sense that each component of $f^{-1}\left(\mathrm{Bd} B^{\prime}\right)$ is a simple closed curve. Let $J^{\prime}$ be an "inner" one of these simple closed curves. Then the interior of the disk bounded by $J^{\prime}$ is mapped by $f$ into Int $B^{\prime}$ or into the interior of some component of $B$. In the latter case, $J^{\prime}$ can be eliminated by making use of the irreducibility of this component of $B$. If $B^{\prime}$ were irreducible, $J^{\prime}$ could be eliminated in the first case also, and one is led after a finite number of steps to the conclusion that $J$ could be shrunk to a point in $B_{0}$. Hence, $B^{\prime}$ is reducible.

Let the disk given by Lemma 1 for $B^{\prime}$ be thickened to form a cube $E$ in $B^{\prime}$ such that $\left(\mathrm{Bd} B^{\prime}\right) \cdot E$ is an annulus ring $A$ in $\operatorname{Bd} E$. Let $B_{1}$ be the component of $\mathrm{Bd} B$ containing $A$. If $A$ does not separate $B_{1}$, define $M$ to be the closed interior of $B_{1}$ plus the 3-cell $E$. If $A$ separates $B_{1}$, then the closed interior of $B_{1}$ plus the 3 -cell $E$ has 2 boundary components, and the closed interior of one of these contains the closed interior of the other. The larger closed interior is here taken to be $M$. In either case, there is a compact 3-manifold $M$ with connected boundary such that $M$ contains $B_{1}$ and possibly some other components of $\mathrm{Bd} B$, the only component of $\mathrm{Bd} B$ which $\mathrm{Bd} M$ meets is $B_{1}$, and the genus of $\mathrm{Bd} M$ is less than the genus of $B_{1}$.

Let $\Sigma$ be the collection of closed surfaces obtained by adding $\mathrm{Bd} M$ to the collection of components of $\mathrm{Bd} B$ and deleting those components of $\mathrm{Bd} B$ which lie in $M$. The closed interiors of the surfaces in $\Sigma$ can be joined by tubes to obtain a 3-manifold with connected boundary of genus less than $N$. By induction, there is a cube with handles $F$ such that $B \subseteq$ Int $F$. The 3-cells $\left\{T_{j}\right\}$ are now added to $F$ to obtain a cube with handles containing $C$. Note that if some $T_{j}$ does not lie in Int $F$ then $F$ may need to be adjusted inside a small neighborhood of $T_{j}$ so that each component of $\left(\mathrm{Bd} T_{j}\right) \cdot(\mathrm{Bd} F)$ is a simple closed curve which separates the two components of $\left(\operatorname{Bd} T_{j}\right) \cdot(\operatorname{Bd} B)$ and these simple closed curves bound mutually exclusive disks on $\mathrm{Bd} F$. This adjustment is done by choosing a "thin," nicely situated 3-cell in $T_{j}$ and projecting it out onto $T_{j}$. This completes the proof.

3. Products of contractible open manifolds. A recent result of $\mathbf{M}$. Brown [1] asserts that a space is topologically $E^{n}$ if it is the sum of an ascending sequence of open subsets each homeomorphic to $E^{n}$. 
Theorem 2. Let $U$ be a $W$-space. Then, $U \times E^{1}$ is topologically $E^{4}$.

Proof. Let $U=\sum_{i=1}^{\infty} H_{i}$, where $H_{i}$ is a cube with handles and $H_{i} \subseteq$ Int $H_{i+1}$. By the above result of Brown, it suffices to show that if $i$ is a positive integer and $[a, b]$ an interval of real numbers $(a<b)$, then there is a 4 -cell $C$ such that

$$
H_{i} \times[a, b] \subseteq \operatorname{Int} C \subseteq C \subseteq U \times E^{1} .
$$

There is a finite graph $G$ in (Int $\left.H_{i}\right) \times\{(a+b) / 2\}$ such that if $V$ is an open set in $U \times E^{1}$ containing $G$, then there is a homeomorphism $h$ of $U \times E^{1}$ onto itself such that $h\left(H_{i} \times[a, b]\right) \subseteq V$. But $G$ is contractible to a point in $U \times E^{1}$. Hence, by Lemma 8 of [4], a 4-cell in $U \times E^{1}$ contains $G$, and the result follows.

Obvious modifications in the proof yield the following:

Theorem 3. Let $U_{1}$ and $U_{2}$ be $W$-spaces. Then $U_{1} \times U_{2}$ is topologically $E^{6}$.

Here, Lemma 8 of [4] is used to produce a 6-cell in $U_{1} \times U_{2}$ containing a 2-polyhedron.

Let $W$ be a contractible open 3-manifold. Theorem 2 states that, modulo the Poincaré Conjecture for dimension $3, W \times E^{1}$ is topologically $E^{4}$. With no special restrictions on $W$, however, one can show:

THEOREM 4. If $W$ is a contractible open $n$-manifold, then $W \times E^{n}$ is topologically $E^{2 n}$.

Proof. Using Brown's result again, and the above techniques, it suffices to show that if $M$ is a compact $n$-manifold with boundary in $W$ and if $C$ is an $n$-cell in $E^{n}$, then the interior of some $2 n$-cell in $W \times E^{n}$ contains $M \times C$. But $C$ can be homeomorphically squeezed into a small neighborhood of some vertex $v$ in its interior, so it is enough to show that the interior of a $2 n$-cell contains $M \times\{v\}$. Now, $M$ has nonempty boundary and so contracts (see [7]) onto a finite $(n-1)$-polyhedron $P$ in $M$. By Lemma 8 of [4], a $2 n$-cell in $W \times E^{n}$ contains $P \times\{v\}$ and the proof is completed by Lemma 2 of [4].

It will be shown elsewhere that there exist uncountably many $W$ spaces, no 2 of which are homeomorphic. Hence:

THEOREM 5. There are uncountably many topologically different 3-manifolds $W$ such that $W \times E^{1}$ is topologically $E^{4}$.

Theorem 4 has the following interesting application. Conner and Floyd [Proc. Amer. Math. Soc. vol. 10 (1959) pp. 354-360] have shown that if $R$ is a positive integer which is not a power of a prime, 
then there exists a contractible open $9 R$-manifold $M^{9 R}$ and a map $f$ of period $R$ on $M^{9 R}$ without fixed points. They do not settle the question of whether $M^{9 R}$ is actually $E^{9 R}$. Theorem 4 , however, gives a map of period $R$ on $E^{18 R}=M^{9 R} \times E^{9 R}$ without fixed points, namely the map $f \times 1$, where 1 is the identity map on $E^{9 R}$. J. M. Kister has recently pointed out how to vary the Conner-Floyd construction to insure that $M^{9 R}$ is $E^{9 R}$ (see this issue of Bull. Amer. Math. Soc., pp. 471-474).

4. Questions. Can Theorem 4 be improved to say that $W \times E^{k}$ is $E^{n+k}$, where $1 \leqq k<n$ ?

Can each $W$-space be embedded in $S^{3}$ ? An example suggested to the author by R. H. Bing would seem to indicate a negative answer.

\section{REFERENCES}

1. M. Brown, The monotone union of open n-cells is an open n-cell, Notices Amer. Math. Soc. vol. 7 (1960) p. 478.

2. J. Glimm, Two cartesian products which are euclidean spaces, Bull. Soc. Math. France vol. 88 (1960) pp. 131-135.

3. D. R. McMillan, Jr., On homologically trivial 3-manifolds, doctoral dissertation, University of Wisconsin, June, 1960, pp. 54-59.

4. J. R. Stallings, Polyhedral homotopy-spheres, Bull. Amer. Math. Soc. vol. 66 (1960) pp. 485-488.

5. - On the loop theorem, Ann. of Math. vol. 72 (1960) pp. 12-19.

6. J. H. C. Whitehead, A certain open manifold whose group is unity, Quart. J. Math. (Oxford) vol. 6 (1935) pp. 268-279.

7. - Simplicial spaces, nuclei and m-groups, Proc. London Math. Soc. vol. 45 (1939) pp. 243-327.

Louisiana State University 